\title{
Anaphylaxis Induced by Glucocorticoids
}

\author{
Stephan M. Erdmann, MD, Faris Abuzahra, MD, Hans F. Merk, MD, \\ Anja Schroeder, MD, and Jens M. Baron, MD
}

Glucocorticoids are frequently used to treat allergic reactions. Therefore, allergic reactions to systemic glucocorticoids in particular are considered most unlikely and are not well known. We report on a 23year-old woman with atopic dermatitis who had an anaphylactic reaction after oral administration of prednisolone. On treatment with epinephrine, antihistamines and volume symptoms resolved. Skin testing with a panel of glucocorticoids showed immediate type reactions to prednisolone, prednisolone hydrogen succinate, prednisone, and betamethasone dihydrogen phosphate. In challenge testing the patient tolerated methyl prednisolone and dexamethasone. There is increasing evidence that true allergic immediate type reactions to glucocorticoids exist. The severity of the reaction can vary from a rash to anaphylaxis. However, a patient sensitized to one or a group of glucocorticoids does not have to refrain from all types of glucocorticoids. Careful challenge testing is by far the best way to select glucocorticoids that are safe for future treatment. Clinicians should be aware that allergic reactions to glucocorticoids can occur and that worsening of symptoms does not always mean treatment failure. ( $\mathrm{J}$ Am Board Fam Pract 2005;18:143-6.)

Since the early 1950s, glucocorticoids have been used extensively for pharmacotherapy. Glucocorticoids are applied topically, orally, or intravenously and, if administered in higher doses, show immunosuppressive, antiproliferative, anti-inflammatory, and antiallergic effects. ${ }^{1}$ The antiallergic properties of glucocorticoids would seem to contradict their capacity to induce allergic reactions. However, a few severe adverse reactions, including life-threatening reactions caused by systemic glucocorticoids, have been reported over the past decades. ${ }^{2-6} \mathrm{Be}-$ cause the risk of anaphylactic reactions caused by glucocorticoids is not taken into consideration by most clinicians, severe complications may occur, particularly in the treatment of status asthmaticus or in anaphylactic reactions attributable to high doses (ie, if increasing symptoms are thought to be caused by insufficient therapy, even higher doses of glucocorticoids may be applied).

We report a patient with an immediate and delayed type hypersensitivity to several glucocorticoids, the diagnostic work-up, including challenge testing, and the options for future treatment with

Submitted, revised, 27 December 2004.

From the Department of Dermatology and Allergology, University Hospital of RWTH Aachen, Aachen, Germany. Address correspondence to: Stephan M. Erdmann, Department of Dermatology and Allergology, University Hospital of RWTH Aachen, Pauwelsstr. 30, 52074 Aachen, Germany (e-mail: serdmann@ukaachen.de). glucocorticoids in patients with glucocorticoid allergy.

\section{Case Report}

A 23-year-old woman was prescribed prednisolone (Decortin $\mathrm{H}$ ) because of worsening of atopic dermatitis. Thirty minutes after taking the first oral dose of $40 \mathrm{mg}$ of prednisolone, she developed palmoplantar paraesthesia, tightness of the chest, dizziness, and nausea, and she finally collapsed. In the emergency department, she was treated with epinephrine, antihistamines, and fluid; however, no glucocorticoid was applied because of the clinical history. Within a few hours all symptoms resolved. Two years before at the dentist's office, the patient had shown the same symptoms, albeit less severe, 15 minutes after prednisolone (Dontisolon) had been applied topically on the oral mucosa for parodontitis. Furthermore, 3 years before, the patient had similar symptoms after intramuscular application of prednisolone hydrogen succinate (Solu Decortin H). The patient had a history of atopic dermatitis, allergic rhinitis, and asthma. She also recalled contact dermatitis after topical treatment with several brands of glucocorticoids.

Skin prick testing of a panel of glucocorticoids showed immediate type reactions to prednisolone, prednisolone hydrogen succinate, prednisone, and betamethasone dihydrogen phosphate after 20 minutes, indicating immediate type hypersensitiv- 
Table 1. Results of Skin Prick Testing with a Panel of Glucocorticoids

\begin{tabular}{ll}
\hline Active Substance & $\begin{array}{c}\text { Results of Skin } \\
\text { Prick Test }\end{array}$ \\
\hline Methylprednisolone & Negative \\
Prednisolone & Positive \\
Prednisolone-21-hydrogensuccinate & Positive \\
Prednisone & Positive \\
Methylprednisolone-21-sodium succinate & Negative \\
Betamethasone dihydrogen phosphate & Positive \\
Hydrocortisone & Negative \\
Dexamethasone & Negative \\
Dexamethasone-21-dihydrogen & Negative \\
phosphate & \\
Triamcinolone & Negative \\
Triamcinolonacetonid & Negative \\
\hline
\end{tabular}

ity (Table). There was no reaction to any of the tested glucocorticoids in 5 healthy control subjects, and testing of all additional ingredients of the 2 culprit drugs had no effect. Patch testing revealed delayed type hypersensitivity to prednisolone, amcinonide, and clobetasol propionate. Because radioallergosorbent assays (RAST) for glucocorticoid allergy demonstrating specific $\mathrm{IgE}$ are not commercially available, a special assay was developed. However, this assay failed to proof any specific serum IgE to prednisolone.

Finally, the patient was challenged with methylprednisolone hydrogen succinate and dexamethasone dihydrogen phosphate intravenously and dexamethasone orally in an intensive care unit. All 3 glucocorticoids were tolerated.

\section{Discussion}

Although systemic glucocorticoids are frequently used worldwide to treat allergic reactions, including anaphylaxis, there is an increasing body of literature indicating that severe allergic or allergic type reactions to glucocorticoids occur despite their antiallergic properties. ${ }^{2-6}$ Our patient with a history of an immediate type reaction to prednisolone and a positive skin test to the culprit agent provides additional evidence that immediate adverse reactions to systemic glucocorticoids do exist. Classifying adverse reactions to glucocorticoids, delayed type allergic reactions (contact dermatitis) after topical application have to be distinguished from immediate type reactions after systemic administration. Whereas contact dermatitis induced by topically applied glucocorticoids is frequently observed, ${ }^{7}$ immediate type hypersensitivity is very rare.

Immediate adverse reactions have been described after oral, intravenous, or intra-articular administration of glucocorticoids. ${ }^{5,8}$ A variety of signs and symptoms of adverse immediate reactions have been reported, including urticaria, pruritus, sneezing, nausea/vomiting, dyspnea, bronchospasm, angioedema, hypotension, decreased consciousness, respiratory arrest, and anaphylaxis, including death. ${ }^{5}$ In most cases the pathomechanism has not been conclusively elucidated, and it is most likely that the pathogenesis is not homogenous. So far, groups of corticosteroids with well defined cross-reactivities as described for topical glucocorticoids could not be found. In some cases of negative skin prick tests, idiosyncrasy similar to reactions to salicylic acid was suspected. ${ }^{9}$ Furthermore, among adverse reactions to glucocorticoids, anaphylaxis induced by additives such as carboxymethylcellulose has also been reported. ${ }^{10}$ However, an increasing number of patients with positive skin tests (prick and intradermal tests) to glucocorticoids suggests that immediate type hypersensitivity to glucocorticoids occurs. ${ }^{3,11-15}$ In most of these cases, no in vitro testing such as the RAST assay was performed to demonstrate specific serum IgE antibodies to the culprit glucocorticoids. In a few cases, however, direct proof of specific IgE to glucocorticoids by the RAST method was successful. ${ }^{6}$ Caduff et $\mathrm{al}^{16}$ published results of voluntary reporting of adverse drug reactions to the national Swiss Drug Monitoring Center; between 1981 and 1999, 14 cases of suspected hypersensitivity reactions after parenteral glucocorticoid application have been reported in about 5 million inhabitants in Switzerland. Considering the rather low yield of voluntary reporting, this study suggests that immediate type reactions to glucocorticoids probably occur more often than has been estimated from recent reviews. ${ }^{5}$

In our patient, prick testing with the culprit drug prednisolone, prednisolone hydrogen succinate, prednisone, and betamethasone dihydrogen phosphate was positive suggesting $\operatorname{IgE}$ mediated allergy. Yet direct proof of specific $\mathrm{IgE}$ to prednisolone by the RAST method was not successful. Poor sensitivity of the RAST method compared with in vivo testing such as skin testing is a well known finding in research of drug allergy and has several reasons. With regard to glucocorticoids it has been specu- 
lated that the frequently observed inability to detect specific serum $\operatorname{IgE}$ to glucocorticoids might occur because the glucocorticoid acts as a hapten, which needs to combine with a protein to form the actual antigen. ${ }^{5}$ Therefore, in vitro tests would need to be directed at detecting antibodies to the steroid-protein conjugate rather than the steroid alone. Furthermore, the technical problem of coupling drugs with poor solubility in the RAST assay may result in molecules that do not show the same allergenic properties before and after conjugation. Although the RAST assay may be helpful in confirming IgE-mediated hypersensitivity, a negative test does not rule out immediate type hypersensitivity.

Because glucocorticoids are poorly soluble, for pharmacotherapy they are coupled with esters especially in the position of $\mathrm{C} 21$ to make them watersoluble for intravenous application. It is interesting that there are a few reports on glucocorticoid allergic patients who tolerated only unconjugated nonesterified corticosteroids in challenge tests ${ }^{6,17}$; by contrast, there is a single report of a patient who had positive skin testing only to glucocorticoids without succinate ester but tolerance of derivatives with succinate ester. ${ }^{18}$ These findings suggest an independent immunologic potential of the esters. ${ }^{6}$

Cross-reactivity has been observed between different systemic glucocorticoids, but the recent classification of cross-reactivity between topical glucocorticoids does not seem to apply for immediate adverse reactions to systemic glucocorticoids. ${ }^{5,7}$ Therefore, in patients with confirmed immediate type allergy to one or several glucocorticoids, it is insufficient to base counseling simply on chemical structure relationship. The best way to identify glucocorticoids that are a safe option for future therapy is by careful challenge testing. We challenged our patient with methylprednisolone hydrogen succinate and dexamethasone dihydrogen phosphate intravenously and dexamethasone orally. All 3 glucocorticoids were tolerated.

\section{Conclusion}

This case report illustrates that clinicians should be aware that allergic reactions in response to systemic glucocorticoid administration can occur. Worsening of symptoms may not always suggest treatment failure, but can also result from an allergic reaction to a glucocorticoid. If immediate type hypersensi- tivity to glucocorticoids is suspected, skin testing is very helpful in confirming allergic reactions and identifying specific agents that will be tolerated in future treatment. However, final administration of a challenge dose in a controlled setting remains the only way to identify a "safe" glucocorticoid for the allergic patient.

We thank Dr. Gerhard Burow, Pharmacia Diagnostics (Freiburg, Germany) and Pharmacia Diagnostics AB (Uppsala, Sweden) for all support carrying out the RAST for prednisolone.

\section{References}

1. Rubin RP. Adrenocortical hormones and drugs affecting the adrenal cortex. In: Modern pharmacology (Craig CR, Stitzel RE, editors). Boston: Little, Brown and Co; 1994. p. 731-45.

2. King RA. A severe anaphylactoid reaction to hydrocortisone. Lancet 1960;12:1093-4.

3. Freedman MD, Schocket AL, Chapel N. Anaphylaxis after intravenous methylprednisolone administration. JAMA 1981;245:607-8.

4. Escribano-Rodriguez MM, Gonzalez-Pol J, MunozBellido FJ, de la Calle-Toral A, Velazquez-Amor E, Conde-Hernandez J. Immediate reaction to methylprednisolone with tolerance of other corticosteroids. Allergy 1997;52:677-78.

5. Kamm GL, Hagmeyer KO. Allergic type reactions to corticosteroids. Ann Pharmacotherapy 1999;33: 451-60.

6. Burgdorff T, Venemalm L, Vogt T, Landthaler M, Stolz W. IgE-mediated anaphylactic reaction induced by succinate ester of methylprednisolone. Ann Allergy Asthma Immunol 2002;89:425-8.

7. Dooms-Goossens A, Morren M. Results of routine patch testing with corticosteroid series in 2073 patients. Contact Dermatitis 1992;26:182-91.

8. Mace S, Vadas P, Pruzanski W. Anaphylactic shock induced by intraarticular injection of methylprednisolone acetate. J Rheumatol 1997;24:1191-4.

9. Dajani BM, Sliman NA, Shubair KS, Hamzeh YS. Bronchospasm caused by intravenous hydrocortisone sodium succinate (Solu-Cortef) in aspirinsensitive asthmatics. J Allergy Clin Immunol 1981; 68:201-4.

10. Patterson DL, Yunginger JW, Dunn WF, Jones RT, Hunt LW. Anaphylaxis induced by the carboxymethylcellulose component of injectable triamcinolone acetonide suspension (Kenalog). Ann Allergy Asthma Immunol 1995;74:163-6.

11. Chapman SC, Loughnan BA, Somerfield SD. Immediate hypersensitivity skin testing in a case of hydrocortisone anaphylaxis: case report. N Z Med J 1979; 90:380.

12. Rao KV, Anderson RC, O'Brien TJ. Successful renal 
transplantation in a patient with anaphylactic reaction to Solu-Medrol (methylprednisolone sodium succinate). Am J Med 1982;72:161-3.

13. Fulcher DA, Katelaris CH. Anaphylactoid reaction to intravenous hydrocortisone sodium succinate: a case report and literature review. Med J Aust 1991; 154:210-4.

14. Murrieta-Aguttes M, Michelen V, Leynadier F, Duarte-Risselin C, Halpern GM, Dry J. Systemic allergic reactions to corticosteroids. J Asthma 1991; 28:329-39.

15. Moreno-Ancillo A, Martin-Munoz F, MartinBarroso JA, Diaz-Pena JM, Ojeda JA. Anaphylaxis to 6-alpha-methylprednisolone in an eight-year-old child. J Allergy Clin Immunol 1996;97:1169-71.

16. Caduff C, Reinhart WH, Hartmann K, Kuhn M. Immediate type hypersensitivity reactions to parenteral glucocorticoids. Schweiz Med Wochenschr 2000;130:977-83.

17. Fernandez S, Reano M, Vives R, et al. 6-Methylprednisolone-induced bronchospasm. Allergy 1997; $52: 780-2$.

18. Mendelson LM, Meltzer EO, Hamburger RN. Anaphylaxis-like reactions to corticosteroid therapy. J Allergy Clin Immunol 1974;54:125-31. 